Revista de Derecho Ambiental. Año V N ${ }^{\circ}$. 
La Revista de Derecho Ambiental, editada por el Centro de Derecho Ambiental de la Facultad de Derecho de la Universidad de Chile, constituye un espacio de exposición y análisis en el plano académico del Derecho Ambiental. Su contenido se presenta a través de doctrina, jurisprudencia y recensiones, abordando diversas materias relacionadas con la gestión, institucionalidad y herramientas de protección ambiental y desarrollo sustentable. En sus páginas se presentan artículos de diferentes autores, en los que se analizan y abordan casos y temas jurídico-ambientales de creciente interés y actualidad.

Directora Responsable

Prof. Valentina Durán Medina

\section{Sub Directora Responsable}

Prof. Pilar Moraga Sariego

\section{Editores Responsables}

Jorge Ossandón Rosales

Antonio Pulgar Martínez

\section{Comité Editorial}

Dra. Verónica Delgado Schneider, Universidad de Concepción

Dr. Juan Carlos Ferrada Bórquez, Universidad de Valparaíso

Dr. Iván Hunter Ampuero, Universidad Austral de Chile

Dr. Alberto Olivares Gallardo, Universidad de Talca

Revista de Derecho Ambiental (en línea)

Centro de Derecho Ambiental

Facultad de Derecho. Universidad de Chile

Pío Nono $1,4^{\circ}$ Piso, Providencia, Santiago de Chile

+562 29785354

cda@derecho.uchile.cl

http://www.derecho.uchile.cl/cda

ISSN 0718-0101

Algunos derechos reservados.

Publicada bajo los términos de la licencia Creative Commons atribución - compartir igual 4.0 internacional

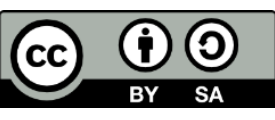




\title{
¿Susurros al viento? Desempeño de la participación ciudadana en el SEIA
}

\author{
Whispers in the wind? Public participation performance in Chile's EIA
}

Ezio Costa*

Abogado, Msc. en Regulación

London School of Economics and Political Science

eziocosta@gmail.com

Victoria Belemmi*

Abogada, U. Chile

belemmi@fima.cl

\begin{abstract}
Resumen: La institucionalidad ambiental ha incorporado a la participación ciudadana como un principio fundamental que contribuye a mejorar las decisiones ambientales, siendo expresión de ello su incorporación en el Sistema de Evaluación de Impacto Ambiental (SEIA). En razón de lo anterior, el presente artículo evalúa el desempeño de la participación ciudadana al interior del SEIA considerándola como una manifestación de la participación ciudadana en la gestión pública. Para ello se toma lo señalado por la Carta Iberoamericana de Participación Ciudadana, para analizar, conforme a la normativa existente y a la práctica jurisprudencial, el procedimiento participativo desde la óptica de tres de sus funciones: comunitaria, pública y privada. Esto, con el objetivo de identificar si es que a más de 20 años desde la entrada en vigencia de la Ley de Bases del Medio Ambiente, se cumple con los objetivos que supone la incorporación de la participación ciudadana en el SEIA.
\end{abstract}

Palabras clave: participación ciudadana, gestión pública, Sistema de Evaluación de Impacto Ambiental, funciones de la participación ciudadana.

Abstract: Environmental regulation considers public participation as a fundamental principle that contributes to improve the environmental decisions, being expression of that its incorporation into the Environmental Impact Assessment System (SEIA). This article

\footnotetext{
* Licenciado en Ciencias Jurídicas y Sociales, Universidad de Chile. Investigador del Centro de Regulación y Competencia (RegCom), Facultad de Derecho, Universidad de Chile y Director Ejecutivo de la ONG FIMA.

* Licenciada en Ciencias Jurídicas y Sociales, Universidad de Chile. Investigadora de la ONG FIMA.
} 
Costa y Belemmi $\bullet$ ¿Susurros al viento? Desempeño de la participación ciudadana en el SEIA $・$ Sección doctrina

evaluates the performance of public participation within this procedure, considering it as a manifestation of public participation in public management. For this purpose, this paper considers the Ibero-American Charter on Public Participation, as a basis to analyze, the participatory procedure from the perspective of three of its functions: community, public and private. The final objective is to identify if the objectives sought at incorporating citizen participation in the SEIA are being achieved, 20 years after it started.

Key words: public participation, public management, Environmental Impact Assessment.

\section{Introducción}

La inquietud por incorporar la participación ciudadana en la toma de decisiones se ha hecho presente desde hace décadas. Chile ha enfocado sus esfuerzos en incorporarla en diferentes esferas públicas, siendo una de las más patentes, la institucionalidad ambiental. Esta última pretende erigir su legitimidad, en gran parte, en el desarrollo de procedimientos de participación ciudadana para la toma de sus decisiones, siendo una manifestación de ello el procedimiento de participación ciudadana (PAC) al interior del Sistema de Evaluación de Impacto Ambiental (SEIA).

En específico, el SEIA partió reconociendo a la participación como uno de sus principios, y luego la consagró como un deber del Estado que si bien comienza con la norma, evoluciona en la comprensión jurisprudencial e incluso en los cambios normativos. Hoy, a más de 20 años de la institucionalidad ambiental, ¿cómo podemos evaluar a la participación ciudadana dentro del SEIA?

Evaluar la participación es una tarea que emprenderemos desde la identificación de ciertas funciones claves en la gestión pública que la literatura le ha reconocido y que revisaremos a continuación. A la luz de estos objetivos iniciales es posible realizar una evaluación respecto al rol que juega la participación ciudadana dentro del SEIA, poniendo especial atención en la particularidad de ser un procedimiento diseñado para aprobar proyectos una vez verificado si se cumple o no con la legislación ambiental. En parte debido a lo anterior, pareciera que la participación ciudadana se ha convertido en el conducto regular para que la ciudadanía manifieste su oposición a ciertos proyectos de inversión, lo que ha hecho que adquiera características especiales. No se trata de un simple diálogo entre la Administración y los ciudadanos que permita nutrir una política púbica, una ley o un reglamento, porque aunque es claro el interés público que hay en juego, en los hechos los intereses del titular y de los observantes son tratados como contrapuestos.

En relación a lo anterior, la pregunta que fluye es relativa a la función que pretende cumplir la participación ciudadana en el procedimiento de evaluación y la manera en que cumple o no con dicha función. En definitiva, el presente artículo contrastará las funciones reconocidas a los mecanismos de participación ciudadana, con las normas y prácticas en el 
SEIA. Este contraste nos permitirá dilucidar cuáles de dichas funciones han sido relevadas y las posibles consecuencias de ello.

\section{La participación ciudadana}

El concepto de participación ciudadana goza de buena fama, pero en general, de poca sustancia. Entendemos que la participación ciudadana es consustancial a la democracia y que en la lógica de la voluntad del pueblo, este derecho es clave. Se le cita permanentemente como un revulsivo frente a las crisis de legitimidad de los gobiernos y se habla de una u otra manera de la importancia de su incorporación en el entramado administrativo, pero la mayoría de las veces, toda esa construcción resulta vacía.

Lo que pasa con la participación ciudadana es que su concepto amplio importa la incorporación de diversos niveles y dimensiones, lo que hace más complejo encontrar conceptos concordantes.

La primera dimensión es aquella que ve a la participación como un principio, cuestión que es especialmente importante en materia ambiental, donde dicho principio opera mayormente. En efecto, el principio participativo fue recogido en el mensaje de la Ley $\mathrm{N}^{\circ}$ 19.300 como necesario "para lograr una adecuada protección del medio ambiente"1, es reconocido por la mayoría de la doctrina nacional en derecho ambiental y es, además, un principio que se hereda directamente de los tratados internacionales suscritos por Chile y que son la base del derecho ambiental.

En esta primera dimensión, Astorga señala que es un principio democrático y que proviene del conocido como principio de cooperación en la doctrina alemana, que supone que la gestión ambiental requiere de la concurrencia de tres actores: las empresas, el Estado y las $\mathrm{ONG}^{2}$. Lo que hace el autor, siguiendo a la lógica alemana, es definir el principio en relación con su función, que es la de lograr una gestión ambiental que vincule a los distintos actores.

En una segunda dimensión, la participación es un derecho, el que se manifiesta como participación política y como participación en la gestión pública. En esta dimensión, normalmente consideramos que la participación está muy vinculada a la idea de democracia y que lo que tanto el ciudadano como el Estado esperan es que se genere cierto espacio de conversación e incidencia.

La participación política es aquella que está vinculada a la operación del sistema político en su conjunto, y por lo tanto al derecho que tenemos los ciudadanos de incidir en dicha operación, ya sea mediante medios directos o indirectos. La participación en la gestión pública, por su parte, mira más a la relación entre los ciudadanos y la Administración

\footnotetext{
${ }^{1}$ Biblioteca del Congreso Nacional, Historia de la Ley 19.300 (Valparaíso: 1993), 17.

${ }^{2}$ Eduardo Astorga, Derecho Ambiental Chileno (Santiago: Lexis Nexis, 2006), 25.
} 
Costa y Belemmi $\bullet$ ¿Susurros al viento? Desempeño de la participación ciudadana en el SEIA $・$ Sección doctrina

del Estado (aunque no está circunscrito a ella) ${ }^{3}$, y es definida por la Carta Iberoamericana de Participación Ciudadana como "el proceso de construcción social de las políticas públicas que, conforme al interés general de la sociedad democrática, canaliza, da respuesta o amplía los derechos económicos, sociales, culturales, políticos y civiles de las personas, y los derechos de las organizaciones o grupos en que se integran, así como los de las comuni-

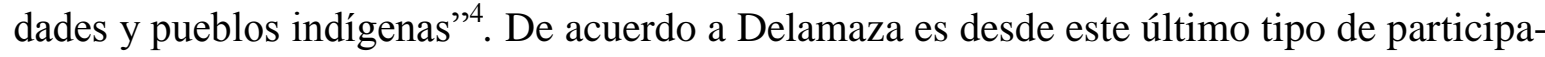
ción que el concepto se construye, siendo por lo tanto habitual que identifiquemos el derecho a la participación con la participación en las políticas públicas ${ }^{5}$ o gestión pública.

La participación ciudadana, además, opera a nivel de mecanismos. Esta dimensión es claramente una bajada de las dos anteriores, en el sentido de que son manifestaciones concretas de un derecho guiado por un principio. Sin embargo, consideramos importante afirmar que esta es una dimensión en sí misma diferente de las anteriores porque es en relación a ella que operan una serie de prácticas, críticas, consideraciones y conceptualizaciones.

Es importante esta mención a la luz de lo que se expresó previamente. Así como el concepto de participación ciudadana suele construirse desde aquella que se realiza en la gestión pública, es común observar una confusión entre el concepto y los mecanismos. Así, identificamos la participación política con el voto (en elecciones de representantes, plebiscitos o referendos) y la participación en gestión pública con cuestiones como las audiencias públicas o la posibilidad de hacer observaciones a una determinada decisión.

Lo anterior representa sin dudas un problema, pues la identificación de un derecho con el mecanismo a través del cual se concretiza produce una distorsión a la hora de evaluar esos mecanismos y su funcionamiento. Es en esta dimensión donde se confunde el derecho a la participación como derecho sustantivo, con el derecho a la participación como derecho procedimental.

En materia ambiental ello es particularmente importante pues se ha entendido que la participación es una de las manifestaciones específicas de la justicia ambiental ${ }^{6}$ y por lo tanto, uno de los contenidos del derecho a vivir en un medio ambiente sano o libre de contaminación. Dicha conceptualización se produce en el cruce con los otros derechos de acceso: el acceso a la información y el acceso a la justicia. En este sentido, Aguilar considera

\footnotetext{
${ }^{3} \mathrm{Al}$ respecto la Carta Iberoamericana de Participación Ciudadana señala en su artículo 17 que: "La creciente provisión de bienes y servicios públicos a través de entidades mercantiles y de organizaciones sociales, obliga a establecer mecanismos de participación ciudadana asociados a la gestión privada o social de servicios públicos transferidos por el Estado.

Las políticas y normas de regulación sobre los bienes y servicios públicos transferidos por el Estado podrán contemplar los mecanismos de participación ciudadana pertinentes a tales formas de gestión." (CLAD, Carta Iberoamericana de participación ciudadana en la gestión Pública (Lisboa: 2009).

${ }^{4}$ CLAD, Cit. Ant. (3), artículo 2.

${ }^{5}$ Gonzalo de la Maza, «Espacio público y participación ciudadana en la gestión pública en Chile: Límites y Posibilidades», Polis. Universidad Bolivariana, vol. $10 \mathrm{~N}^{\circ} 30$ (2011): 47.

${ }^{6}$ Dominique Hervé, Justicia Ambiental y Recursos Naturales (Valparaíso: Ediciones Pontificia Universidad Católica de Valparaíso, 2015), 66.
} 
que los derechos de acceso son, por una parte, un requisito del derecho a vivir en un medio ambiente sano, y por otra, se fortalecen recíprocamente en aras a conseguir la justicia en materia ambiental, ya que los tres derechos tendrían tanto una vertiente procesal como una material relacionada con el derecho a vivir en un medio ambiente sano ${ }^{7}$.

La participación ciudadana en el SEIA es en principio participación en la gestión pública, y se lleva a cabo con un mecanismo muy específico como es el que la propia Ley $\mathrm{N}^{\circ} 19.300$ ha creado. Este mecanismo, si bien acepta distintas metodologías, es finalmente consultivo e incorpora la obligación para el Estado de considerar las observaciones que se hayan formulado, además de otorgar al observante legitimación activa para reclamar respecto de las resoluciones de calificación ambiental (RCA) cuando sus observaciones no se hayan considerado adecuadamente. En el siguiente acápite se describirá con mayor detalle este mecanismo, pero valga la advertencia de situarnos en esta dimensión de la participación.

El hecho de que consideremos la participación ciudadana en el SEIA como un mecanismo de participación en la gestión pública, importa entender que dicho mecanismo debe cumplir con ciertos estándares mínimos para poder considerar que se está respetando el derecho a la participación y se está honrando el principio participativo. Como veremos, algunos de esos estándares han sido expresados o esbozados en la Ley $\mathrm{N}^{\circ}$ 19.300, mientras que otros fluyen de otras fuentes.

\section{La participación ciudadana en la Ley $\mathbf{N}^{\circ} 19.300$}

El reconocimiento de la participación ciudadana en materia ambiental se encuentra en diversos instrumentos internacionales, siendo uno de los más importantes el principio 10 de la Declaración de Río sobre el Medio Ambiente y el Desarrollo del año 1992, en el que se reconoce: i) el derecho a acceder a la información ambiental; ii) el derecho a participar directamente en la toma de decisiones, entregando a los Estados el deber de facilitar y fomentar ambos derechos; y ii) el derecho de acceso a la justicia ambiental ${ }^{8}$.

Es a la luz de esta Declaración que en Chile se creó la institucionalidad ambiental y la Ley $\mathrm{N}^{\circ} 19.300$ de Bases Generales del Medio Ambiente, la que contempló el principio

\footnotetext{
${ }^{7}$ Gonzalo Aguilar, Gobierno Ambiental por consentimiento, participación y evaluación ambiental. En: Evaluación medioambiental, participación y protección del medio ambiente (Santiago: Librotecnia, 2013), 128.

${ }^{8}$ En específico el Principio 10 de la Declaración de Río señala que: "El mejor modo de tratar las cuestiones ambientales es con la participación de todos los ciudadanos interesados, en el nivel que corresponda. En el plano nacional, toda persona deberá tener acceso adecuado a la información sobre el medio ambiente de que dispongan las autoridades públicas, incluida la información sobre los materiales y las actividades que encierran peligro en sus comunidades, así como la oportunidad de participar en los procesos de adopción de decisiones. Los Estados deberán facilitar y fomentar la sensibilización y la participación de la población poniendo la información a disposición de todos. Deberá proporcionarse acceso efectivo a los procedimientos judiciales y administrativos, entre éstos el resarcimiento de daños y los recursos pertinentes".
} 
participativo como uno de sus pilares. En esa línea es que la Ley $\mathrm{N}^{\circ} 19.300$ reconoció a la participación ciudadana y señaló en su artículo 4 como un deber estatal su facilitación. Vale decir, de las dimensiones antes tratadas, la Ley $\mathrm{N}^{\circ} 19.300$ primero que todo viene informada por el principio de participación, lo que se manifiesta en el mensaje de la ley antes citado, y enseguida, reconoce el derecho a la participación y lo hace además desde su faz prestacional, al establecer claramente la obligación que tiene el Estado a través de sus organismos, de facilitar dicha participación.

Tal deber se ve concretado por el artículo 83 del Decreto 40/2012 del Ministerio del Medio ambiente, Reglamento del SEIA, el que señala como obligaciones directas del Servicio de Evaluación Ambiental (SEA) a través de las Comisiones de Evaluación o del Director del Servicio "establecer los mecanismos que aseguren la participación informada de la comunidad". Es así como al SEA le corresponde generar los mecanismos necesarios para que la participación ciudadana pueda llevarse a cabo de forma efectiva, debiendo evaluar las condiciones particulares de cada comunidad para establecer los medios más idóneos.

El hecho de que la Ley $\mathrm{N}^{\circ} 19.300$ reconozca el derecho a la participación desde su dimensión positiva y prestacional, importa una diferencia a la hora de intentar comprender el contenido del mismo en materia ambiental, pues si bien esta dimensión positiva es propia del derecho mismo, independientemente de la materia que se trate, en este caso contamos con un mandato explícito que no surge de manera evidente para todos sus instrumentos de la participación ciudadana en la gestión pública, toda vez que permite participar tanto en las propuestas que tienen su origen en la Administración (por ejemplo para la determinación de una Norma de Calidad Ambiental ${ }^{9}$ ) como en las decisiones que implican referirse a propuestas que son creadas por privados, y que son sometidas a evaluación por la Administración mediante el SEIA.

Y esto último es importante porque es un reconocimiento de que la participación de la ciudadanía al interior del SEIA, en un acto de la Administración que implica la relación entre esta última y un particular distinto de quienes tienen derecho a participar, contempla, en primer lugar, la dimensión de derecho a participar en la gestión pública, en cuanto ella opera de forma que la ciudadanía organizada y las personas puedan influir en las decisiones que toma la autoridad ambiental a nivel de Administración, incorporando en dichas deci-

\footnotetext{
${ }^{9}$ La Ley $\mathrm{N}^{\circ} 19.300$ establece mecanismos de participación ciudadana que operan en relación con diferentes instrumentos de gestión ambiental. Cabe recordar en este sentido que la regulación ambiental chilena está organizada principalmente desde esta configuración administrativa de los instrumentos de gestión, siendo ellos los protagonistas en la materia. En este sentido, además del mecanismo de participación en el SEIA, la Ley establece procedimientos de participación ciudadana en la creación de normas de emisión y de calidad (artículo 32, Ley $\mathrm{N}^{\circ}$ 19.300) y en los procedimientos de creación de planes de prevención y descontaminación (artículo 44, Ley $\mathrm{N}^{\circ}$ 19.300). En ambos casos, la Ley ha mandatado la creación de reglamentos que contengan algunas etapas procedimentales mínimas y entre ellas "una adecuada publicidad" y la "consulta a organismos competentes públicos y privados", de lo cual los reglamentos en cuestión, en una correcta interpretación a la luz del derecho a la participación, han derivado procedimientos que contienen participación ciudadana abierta, aunque no exenta de dificultades que no son objeto del presente artículo.
} 
siones su información y preocupaciones, y contempla, en segundo lugar, una dimensión que va más allá de la concreción del derecho a la participación en sí mismo y su cooperación con la Administración, toda vez que hace transparente su vinculación a la protección del derecho a vivir en un medio ambiente libre de contaminación.

\section{La participación ciudadana en el SEIA}

En lo que nos interesa en este trabajo y que respecta al SEIA, la Ley $\mathrm{N}^{\circ} 19.300$ estableció una instancia de participación ciudadana en sus artículos 26 y siguientes, con ciertas particularidades iniciales que luego fueron variadas por la modificación realizada a la institucionalidad ambiental del año 2010.

En principio la participación en el SEIA, i) solo era procedente respecto de proyectos ingresados por Estudios de Impacto Ambiental; ii) solo se contemplaba una vez respecto del proyecto, sin existir la posibilidad de iniciar un nuevo procedimiento ante modificaciones sustanciales; iii) solo era procedente respecto de organizaciones con personalidad jurídica y de personas naturales directamente afectadas; y iv) se contemplaba solo una reclamación administrativa en contra de las RCA cuando las observaciones realizadas no hubiesen sido "debidamente ponderadas".

Tales particularidades provocaron que el SEIA fuese blanco de diversas críticas, incluyendo las realizadas por la OCDE en el año 2005, en su Informe de Desempeño Ambiental, en el cual se señaló que:

"Sin embargo, en lo relativo a la información ambiental, se deben consolidar y regularizar las labores relativas a los datos ambientales, los informes de medio ambiente y los indicadores ambientales. Se debe dar continuidad al desarrollo del SINIA para integrar la información sectorial, mejorar la calidad de la información ambiental física e incluir información económica sobre el medio ambiente (gasto en medio ambiente, empleo en el sector ambiental, precios del agua, entre otros). La práctica y los mecanismos de participación pública, que avanzaron durante el período examinado, deberán continuar mejorando para ser más eficientes y sistemáticos, a nivel nacional y regional, particularmente en asociación con las EIA de proyectos y las evaluaciones ambientales estratégicas (EAS) de las políticas, planes y programas públicos" $"$.

El informe antes citado, destacó los avances en materia de información ambiental y de participación ciudadana, sin embargo, recomendó continuar reforzando las instancias participativas al interior del SEIA. Teniendo en cuenta ello y el desarrollo práctico, es que entre las modificaciones introducidas en el año 2010 por la Ley $\mathrm{N}^{\circ} 20.417$, se consideraron algunas mejoras en materia de participación ${ }^{11}$.

En específico, se incluyó en el artículo 30 bis, un proceso de participación ciudada-

\footnotetext{
${ }^{10}$ OCDE-CEPAL, Evaluación de Desempeño Ambiental (Santiago, 2005), acceso el 30 de agosto de 2017, http://repositorio.cepal.org/bitstream/handle/11362/1288/S0500003_es.pdf;jsessionid=20A3F661EB0CE290E 3F449ECDC1BC7CB? sequence=1, 30 .

${ }^{11}$ CEPAL, Acceso a la información, participación y justicia en temas ambientales en América Latina y el Caribe. Situación actual, perspectivas y ejemplos de buenas prácticas. Serie Medio Ambiente y Desarrollo $N^{\circ}$ 151 (Santiago, 2013), acceso el 31 de agosto de 2017, http://www.cepal.org/publicaciones/xml/9/51389/Accesoalainformacion.pdf, 13.
} 
Costa y Belemmi $\bullet$ ¿Susurros al viento? Desempeño de la participación ciudadana en el SEIA $・$ Sección doctrina

na por un plazo de 20 días, respecto de aquellos proyectos que siendo presentados por Declaraciones de Impacto Ambiental (DIA) generen cargas ambientales, debiendo ser solicitado por al menos dos organizaciones ciudadanas con personalidad jurídica o por diez personas naturales directamente afectadas.

Además, la Ley $\mathrm{N}^{\circ} 20.417$ se hizo cargo de la crítica realizada a la institucionalidad en cuanto a la imposibilidad de activar instancias de participación ciudadana luego de la instancia inicial, toda vez que las modificaciones realizadas al proyecto en el procedimiento redundaban en que lo aprobado nada tuviese que ver con lo inicialmente presentado, respecto de lo cual se generaba la participación. Es por ello que se incorporó al artículo 29 de la Ley $\mathrm{N}^{\circ} 19.300$ el actual inciso segundo, el que permite abrir una nueva etapa de participación ciudadana, por 30 días, cuando el proyecto hubiese sido objeto de modificaciones sustanciales respecto de lo inicialmente presentado. Asimismo, respecto de las modificaciones sustanciales en las DIA se contempló en el artículo 30 bis la posibilidad de abrir una nueva instancia de participación ciudadana por 10 días.

Finalmente, otras dos modificaciones importantes realizadas por la Ley $\mathrm{N}^{\circ} 20.417$ respecto de la participación ciudadana al interior del SEIA, son la ampliación que realiza respecto de quienes pueden participar, ya que extiende la legitimidad a cualquier persona natural o jurídica y la posibilidad de reclamar en contra de la RCA cuando las observaciones ciudadanas "no hubieren sido debidamente consideradas" tanto ante la administración como ante los tribunales de justicia.

Con estas modificaciones (y varias otras no centrales a este trabajo) se pretendió dar un nuevo impulso a la institucionalidad ambiental, con el fin de alcanzar los estándares internacionales en la materia.

Desde la dictación de la Ley $\mathrm{N}^{\circ} 19.300$ han transcurrido más de 20 años, mientras que desde las modificaciones a la institucionalidad realizadas por la Ley $\mathrm{N}^{\circ} 20.417$ han transcurrido 7 años, siendo posible evaluar si es que efectivamente se está otorgando el lugar central que merece la participación ciudadana.

En este sentido, es importante considerar lo que la nueva Evaluación de Desempeño Ambiental (2005-2016) de la OCDE ha señalado, siendo del caso que el organismo internacional reconoce avances en materia de institucionalidad ambiental pero pone dentro de sus grandes pendientes, precisamente, a la participación ${ }^{12}$.

En lo que se refiere en específico al SEIA, la OCDE considera que: "Sin embargo, las disposiciones vigentes sobre participación de la ciudadanía en estas evaluaciones no contienen disposiciones que garanticen la adecuada consideración de proyectos alternativos o la minimización del impacto ambiental potencial, lo que puede dar origen a conflictos ambientales y sociales"13.

Como es posible observar de lo señalado por la OCDE y veremos además en lo sucesivo, si bien las modificaciones introducidas por la Ley $\mathrm{N}^{\circ} 20.417$ son relativamente significativas, ello no ha significado dar cabida de manera completa al derecho a la participación, ni una mejora sustantiva en la forma en que la participación opera en el SEIA.

\footnotetext{
${ }^{12}$ OCDE-CEPAL, Evaluación del Desempeño Ambiental de Chile (Santiago: 2016), acceso el 31 de agosto de 2017, http://repositorio.cepal.org/bitstream/handle/11362/40308/S1600413_es.pdf, 18.

${ }^{13}$ OCDE-CEPAL, Cit. Ant. (12), 29.
} 


\section{Funciones de la participación ciudadana}

La participación ciudadana carece de un concepto unívoco, pero una de las cuestiones en que generalmente podemos encontrar coincidencias es en su comprensión funcional, que ve a la participación precisamente desde las funciones que este derecho y sus herramientas representan. En estas visiones, son dichas funciones las que, teóricamente, hacen deseable a la participación en un Estado de Derecho.

En este sentido, la Carta Iberoamericana de Participación Ciudadana en la Gestión Pública, a la que Chile suscribió en el año 2009, indica en sus artículos 3, 4 y 5, algunas de dichas funciones. En específico señala que: i) es consustancial a la democracia; ii) contribuye a la gobernabilidad; iii) refuerza la posición activa de los ciudadanos y las ciudadanas como miembros de sus comunidades; iv) permite la expresión y defensa de sus intereses, el aprovechamiento de sus experiencias y la potenciación de sus capacidades, contribuyendo de esta manera a mejorar la calidad de vida de la población; v) fomenta una nueva cultura, en la que la ciudadanía va adquiriendo una mayor disposición a informarse acerca de los asuntos públicos, a cooperar y a respetar la diversidad social y cultural; y vi) ayuda a abordar los conflictos y a propiciar acuerdos ${ }^{14}$.

Como vemos, hay un primer grupo de funciones, al que denominaremos comunitarias, que tiene que ver con la vida en comunidad y la dimensión social de la participación, donde lo importante es el hecho de que la misma participación permita desarrollar o densificar el tejido social. Un segundo grupo de funciones, al que denominaremos privadas, está relacionado con los intereses privados, tanto de las personas que participan como eventualmente de quien se ve afectado por dicha participación. En este segundo grupo de funciones, lo relevante es que existen intereses particulares que en algunos casos serán transables y posibles de satisfacer mediante negociación u otros mecanismos del tipo.

Finalmente, existe una función propiamente pública de la participación, que la Carta Iberoamericana identifica con la legitimidad y la efectividad de las decisiones. En ese sentido, Castellá, por ejemplo, indica que con la participación ciudadana se estaría permitiendo que los ciudadanos se incorporen a la democracia de modo directo ${ }^{15}$. Por su parte, Larios reconoce también a la legitimidad como una de las funciones de la participación ${ }^{16}$. Por último, Mirosevic ha hecho hincapié en estas funciones públicas y en la prevención de conflictos dentro del SEIA que dicha función importaría ${ }^{17}$.

Es desde estas tres funciones entonces que, en lo sucesivo, este artículo evaluará la manera en que la participación ciudadana es practicada en el SEIA.

Esperamos encontrar cómo y cuánto la herramienta de participación en el SEIA propende a cada una de las funciones antes determinadas. Para evaluar lo anterior se toma-

\footnotetext{
${ }^{14}$ CLAD, Cit. Ant. (3), artículos 3, 4 y 5.

15 Josep Castellá, Los derechos constitucionales de participación política en la Administración Pública (Barcelona: Cedecs, 2011), 147-148.

${ }^{16}$ María Jesús Larios, La participación ciudadana en la elaboración de la ley (Madrid: Congreso de los diputados, 2003), 302-312.

${ }^{17}$ Camilo Mirosevic, «La participación ciudadana en el procedimiento de evaluación de impacto ambiental y las reformas introducidas por la Ley N²0.417», Revista de Derecho de la Pontificia Universidad Católica de Valparaíso 36 (2011): 285.
} 
rán en consideración principalmente dos cosas; i) documentos elaborados por el SEA en los que se contengan recomendaciones o regulaciones relativas a la manera de llevar adelante procesos de participación ciudadana en el SEIA, y ii) resoluciones judiciales que se hayan referido a procedimientos de participación ciudadana en el SEIA.

Sin perjuicio del análisis que realizaremos, nos parece que no existe una práctica al interior del SEIA que sea manifestación de solo una función, toda vez que estas se interrelacionan y se encuentran presentes en cada una de las actividades con mayor o menor fuerza. Por ello, mejorar una deficiencia del sistema en relación a la participación ciudadana implica contribuir al cumplimiento de todas las funciones en su conjunto y las situaciones elegidas son meramente ejemplificadoras.

\subsection{Función comunitaria}

Conforme se indicó, hemos denominado función comunitaria aquella que da cuenta de que la participación ciudadana sirve para reforzar la posición de los ciudadanos en la comunidad y para fomentar una cultura de cooperación y respeto a la diversidad, potenciando la vida en comunidad. Para que ello sea efectivo, lo que la comunidad tenga que decir respecto a la instalación de un proyecto debiera verse reflejado en la decisión final.

Podemos sostener que la Ley $\mathrm{N}^{\circ} 19.300$ reconoce este elemento, como se evidencia en el Mensaje de la Ley al expresar: "El principio de la participación se puede apreciar en muchas de las disposiciones. En primer término, procurando que las organizaciones locales puedan informarse y, en definitiva, hasta impugnar los nuevos proyectos en proceso de autorización por causar un impacto ambiental significativo y negativo sobre el ambiente. Se pretende que terceros distintos de los patrimonialmente afectados puedan accionar para proteger el medio ambiente, e incluso obtener la restauración del daño ambiental"18.

Además, la reforma incorporada por la Ley $\mathrm{N}^{\circ} 20.417$ significó un avance sustantivo en el reconocimiento de las comunidades en materia de participación, al entregar la posibilidad de que cualquier persona jurídica o natural pueda realizar observaciones ciudadanas. En línea con el Principio 10 de la Declaración de Río, tal posibilidad además es reforzada por la consagración del acceso a la información pública y por una acción que permite a quienes hicieron observaciones ciudadanas acudir a los tribunales de justicia en caso de que las observaciones realizadas en el procedimiento no sean "debidamente consideradas" en los fundamentos de la decisión.

No obstante lo anterior, es posible identificar al menos dos elementos relacionados que merman el derecho a la participación ciudadana de la comunidad: i) la indefinición de la Ley respecto a la "debida consideración de las observaciones ciudadanas"; y ii) las asimetrías de información existentes entre la comunidad, Administración y titular.

Respecto a lo primero, ni la Ley ni su reglamento han definido qué se entiende por observación ciudadana y cuándo esta se encuentra debidamente considerada, lo que ha significado que los únicos dos instructivos referidos a la participación ciudadana disponibles del SEA se refieran a este tópico.

\footnotetext{
${ }^{18}$ Biblioteca del Congreso Nacional, Cit. Ant. (1), 17.
} 
El Oficio Ordinario $N^{\circ} 100.142$ del SEA, que instruyó sobre la admisibilidad de las observaciones ciudadanas al interior del SEIA, muestra amplitud a la hora de declarar admisible una observación ciudadana, estableciendo que se entenderá por ella: "toda opinión, comentario, pregunta y/o inquietud formulada por una persona, a fin de conocer, advertir, reparar o manifestarse acerca de las características de un proyecto o actividad, su línea de base, los impactos ambientales que presenta o genera y las medidas de mitigación, reparación y compensación propuestas, considerando la información disponible en el respectivo Estudio de Impacto Ambiental" 19 .

Se concluye con que los únicos requisitos para que una observación sea admisible, es que ésta sea presentada por escrito y dentro de plazo, y contenga nombre y domicilio, pudiendo presentarla en papel o mediante la plataforma electrónica dispuesta para ello ${ }^{20}$.

Por su parte, el Oficio Ordinario $\mathrm{N}^{\circ} 130.528$ del año 2013, vino a realizar definiciones sobre qué se debe entender por "debida consideración" de las observaciones ciudadanas, indicando los criterios para determinar cuándo una observación se considera pertinente. Así el instructivo señala que "considerar" debe entenderse como "hacerse cargo de la materia observada" con el fin de dar respuesta a los requerimientos de la ciudadanía. Agrega que será pertinente toda observación que tenga carácter ambiental, y que la "consideración será de carácter técnico y se efectuará en función de lo que está consignado en el expediente de evaluación, atendiendo de manera clara y precisa al requerimiento" ${ }^{, 21}$.

La participación ciudadana al interior del SEIA es de carácter consultivo, y en ese sentido no existe una obligación para la Administración de vincular su decisión final a lo observado por los participantes. No obstante ello, sí se encuentra obligada a i) responder y ii) considerar debidamente las observaciones realizadas que sean pertinentes, lo que implica que tengan influencia real en las decisiones que tome la Administración, cuando correspon$\mathrm{da}^{22}$.

Si bien el estándar legal pareciera ser alto, de lo señalado en el instructivo se evidencia que a falta de contenido legal o reglamentario que venga a definir qué significa "debidamente consideradas en los fundamentos" la consagración ha perdido fuerza y la Administración (el SEA) ha profundizado esa debilidad. Esto redunda en que las observaciones realizadas no tengan real influencia en la decisión final, toda vez que el Servicio ha interpretado la forma de considerarlas como responder de acuerdo a lo que el titular ha entregado en el expediente, cuestión que es insuficiente y que no tiene relación con el derecho a la

\footnotetext{
${ }^{19}$ Servicio de Evaluación Ambiental, Oficio Ordinario $N^{\circ}$ 100.142: Instruye sobre la admisibilidad de observaciones ciudadanas en los procesos de participación ciudadana en el SEIA (Santiago, 2010), Numeral 2, 1.

${ }^{20}$ Servicio de Evaluación Ambiental, Cit. Ant. (19), Punto 7, 4.

${ }^{21}$ Servicio de Evaluación Ambiental, Oficio Ordinario $N^{\circ} 130.528$ : Imparte instrucciones sobre la consideración de las observaciones ciudadanas en el marco del procedimiento de evaluación de impacto ambiental. (Santiago: $1^{\circ}$ de abril de 2013), Numeral 2.3 letra a).

${ }^{22}$ En ese sentido se ha manifestado el Segundo Tribunal Ambiental en su reciente fallo en la causa Rol R131-2016, de fecha 28 de abril del año 2017, ha indicado que: "Vigésimo cuarto. Que, en todo caso, cabe aclarar que la debida consideración de las observaciones ciudadanas, por parte de la Administración, obviamente no implica concluir en una posición favorable a lo observado, pero sí obliga a la autoridad a motivar adecuadamente su respuesta, no siendo suficiente una mera transcripción de las opiniones del titular o de los organismos sectoriales, sino que deberá contener una revisión acuciosa de todos los elementos tenidos en cuenta en la evaluación vinculados a la preocupación ambiental objeto de la observación".
} 
Costa y Belemmi $\bullet$ ¿Susurros al viento? Desempeño de la participación ciudadana en el SEIA $・$ Sección doctrina

participación ciudadana, ya que las respuestas son apenas una especie de información concentrada, de bajo valor y nulo impacto.

El segundo elemento, muy ligado a lo anterior, es la dificultad a la que se enfrentan quienes quieren participar, debido al volumen y a lo compleja y técnica que es la información contenida en los expedientes. Aquellos componentes dificultan la comprensión del proyecto y de las respuestas entregadas por el SEA constatándose asimetrías de información. Sobre todo en consideración a que, muchas veces, el estándar discutido sobre las reclamaciones administrativas y judiciales de estas pretenden una exactitud impropia, olvidando que las observaciones ciudadanas abren temas a discutir que no pueden ser medidos con estándares técnicos estrictos, toda vez que eso atenta contra el principio de participación ciudadana, excluyendo a quienes no poseen los conocimientos científicos adecuados. Ello ha sido evidenciado por la Comisión Asesora Presidencial para la Evaluación del SEIA, la que en su informe final del año 2016, indicó que: "no existe el espacio ni la posibilidad para que las comunidades puedan incidir en los aspectos que resultan de su interés. Esta imposibilidad de incidencia, sumado a problemas de asimetría de la información entre titulares, ciudadanía y el Estado, ha llevado a que la participación ciudadana en el SEIA pierda prestancia y legitimidad ante las comunidades y, al mismo tiempo, ha propiciado una crítica negativa al SEA respecto de su gestión como institución del Estado"23.

Por lo mismo, que el instructivo ( $\mathrm{N}^{\circ}$ 130.528) aplicable a las observaciones ciudadanas señale como premisa que será técnica y basada en lo que se indica en el expediente, perpetúa el déficit y mantiene las asimetrías de información. En efecto, se espera que la observación realizada sea debidamente procesada, que se analice y que en base a ello se confeccione una respuesta que no necesariamente resulte exactamente coincidente con la información entregada por el titular ${ }^{24}$.

Esto es abiertamente contrario a uno de los principios de la participación ciudadana definidos por la OCDE, cual es el de claridad (el que se encuentra recogido por el Instructivo). De acuerdo a este principio, y como es evidente, la participación ciudadana debiera hacerse lo más clara y simple posible para las personas, expresando cómo se lleva adelante y quiénes serán los responsables ${ }^{25}$. En este sentido se ha manifestado el Consejo Nacional de Participación Ciudadana y Fortalecimiento de la Sociedad Civil, el que en su informe final expresa como una de las necesidades que la participación se lleve adelante en un "entorno favorable", vale decir, accesible a los ciudadanos e inclusivo para personas en diferentes situaciones, de diferentes niveles culturales y educacionales, pueblos originarios y colectividades $^{26}$.

\footnotetext{
${ }^{23}$ Ministerio del Medio Ambiente, Comisión Asesora Presidencial para la Evaluación del SEIA. Informe Final (Santiago: Junio 2016), acceso el 31 agosto 2017, http://portal.mma.gob.cl/wpcontent/doc/35877_Informe-MMAF_FINAL.pdf

${ }^{24}$ En ello la posibilidad que los organismos sectoriales tengan que incorporar las observaciones ciudadanas tiene mucho que aportar. Por ello una de las críticas al SEIA es la diferencia de plazos con que cuentan los organismos para referirse a lo presentado por el titular y por los observantes, versus el plazo extensible con que cuenta el titular para responder a las observaciones realizadas por la Administración.

${ }^{25}$ OECD, Focus on Citizens: Public Engagement for Better Policy and Services (París, 2009), acceso el 31 agosto 2017, http://www20.iadb.org/intal/catalogo/pe/2009/03785.pdf, 17.

${ }^{26}$ Consejo Nacional de Participación Ciudadana, Estado de la participación ciudadana en Chile y propuestas de reforma a la Ley 20.500 sobre Asociaciones y Participación Ciudadana en la Gestión Pública (Santiago:
} 
Incluso el propio reglamento del SEIA se preocupó de expresar, al menos desde el punto de vista de la entrega de información, esta necesidad. Así el artículo 83, inciso $5^{\circ}$ señala: "Asimismo, el Servicio propiciará la instancia de encuentro entre el titular y la comunidad, con el objeto que ésta se informe sobre las particularidades del proyecto o actividad. Estas actividades deberán realizarse oportunamente en un lenguaje sencillo, directo y de fácil comprensión para la comunidad. De estas actividades deberá quedar constancia en el expediente".

Como se puede apreciar, en el SEIA existen herramientas que permiten a la comunidad hacerse parte del proceso y recibir respuesta, existiendo además la obligación de que ello sea hecho de forma comprensible e inclusiva. Sin embargo, la práctica no ha podido superar las asimetrías de información y la incidencia de la comunidad en la decisión final ha quedado circunscrita a la capacidad técnica que fortuitamente pueda tener una comunidad o no. Con ello, sostenemos que el sistema se encuentra claramente en deuda con el cumplimiento de la función comunitaria de la participación ciudadana.

No obstante lo anterior, el Segundo Tribunal Ambiental ha sostenido en sus recientes fallos la importancia del principio participativo, intentando dar contenido más específico al mismo. En su fallo en la causa Rol R-35-2014, en el que se decidió dejar sin efecto la RCA por afectación de la participación ciudadana ${ }^{27}$, se hace cargo de los estándares señalados en el Instructivo 130.528 y señala que si la "la información o su disponibilidad fue insuficiente [...] será imposible asegurar que hubo debida consideración de las observaciones ciudadanas, lo que constituirá un vicio de tal entidad, que sólo es corregible con la correspondiente declaración de nulidad"28. Además, indica que "la 'debida consideración' de la observación, obviamente no implica concluir en una posición favorable a lo observado, pero sí obliga a la autoridad a motivar adecuadamente su respuesta, no siendo suficiente una mera descripción que se limite únicamente a la reproducción de las opiniones del titular o de los organismos sectoriales, sino que deberá contener una revisión acuciosa de todos los elementos tenidos en cuenta en la evaluación" 29.

Conforme a ello, concluye que: "para determinar si las observaciones ciudadanas fueron debidamente consideradas, el análisis se debe extender a todo el procedimiento de evaluación ambiental y no debe quedar circunscrito únicamente a la respuesta que de ella se haga en la RCA respectiva. Tan importante como la respuesta a las observaciones, es el tratamiento que la autoridad les haya dado durante todo el proceso de evaluación antes de dar respuesta formal, donde la autoridad tiene el deber de incorporar a dicha evaluación, con la mayor antelación posible, las observaciones de la ciudadanía, lo que le permitirá adoptar, si corresponde, decisiones oportunas que también constituyen una expresión de una debida consideración de ellas" ${ }^{30}$.

Enero 2017), acceso el 30 agosto 2017, http://consejoparticipacion.cl/content/uploads/2017/01/informefinal.pdf, 54-55.

${ }^{27}$ El mismo argumento fue sostenido por el Segundo Tribunal Ambiental, en el fallo en su causa Rol R-1012016 y en la causa Rol R-131-2016, 28 de abril de 2017.

${ }^{28}$ Segundo Tribunal Ambiental, Rol R-35-2014, 18 de febrero de 2016, Considerando Quincuagésimo Segundo.

${ }_{29}^{29}$ Segundo Tribunal Ambiental Cit. Ant. (28), Considerando Quincuagésimo Tercero.

${ }^{30}$ Segundo Tribunal Ambiental, Cit. Ant. (28), Considerando Quincuagésimo Noveno. 
En esta decisión, el Segundo Tribunal Ambiental da cuenta de la importancia de incorporar a la participación ciudadana en el SEIA, otorgándole un lugar central dentro del procedimiento. Es evidente que el ánimo del tribunal es señalar que la consideración de la observación ciudadana no se circunscribe a la mera respuesta, sino que debe fluir de todo el expediente administrativo, porque solo así es posible dar cuenta de la incorporación de las inquietudes de la comunidad a la decisión final. En este sentido Moraga, refiriéndose al mismo fallo citado, sostiene que: "el trabajo jurisprudencial del Tribunal Ambiental de Santiago deja de manifiesto que la participación es más que un requisito procedimental de la evaluación de impacto ambiental, pues esta persigue incorporar de manera real a los terceros en el proceso de toma de decisiones ambientales, en el sentido en que lo propone el desarrollo sustentable" 31 .

Finalmente, si bien la posición sostenida por el tribunal implica un gran avance en la definición de la participación ciudadana como principio y derecho, cabe consignar que en una interpretación reduccionista y voluntarista, se puede también entender que, en principio, bastaría con reinterpretar el expediente administrativo para saber si se consideró la observación, no dando importancia al deber de dar respuestas claras y suficientes. Esta interpretación dejaría en peor posición al observante, pues de tener una respuesta que concentra información, podría pasar a no tener una respuesta directa, cuestión que luego permitiría al SEA argüir que la consideración de una observación fluye del expediente administrativo.

Hemos dejado fuera del análisis una de las dimensiones de esta función que puede resultar crucial, pero que lamentablemente es inabordable metodológicamente por un trabajo como este, que es la capacidad que tienen las instancias de participación ciudadana relativa a proyectos, de constituirse en espacios en que la comunidad se articule en relación con intereses de mayor envergadura. No obstante ello, cabe consignar que ya en el año 2003, Rojas, Sabatini y Sepúlveda relevaban la importancia de la articulación de la ciudadanía a instancias del SEIA, indicando en específico que "para los ciudadanos y comunidades locales, los conflictos ambientales han sido oportunidades de fortalecimiento de su organización, de su identidad y de sus proyectos" ${ }^{32}$ lo que ha redundado el fortalecimiento de la sociedad civil y en una mayor injerencia en las decisiones políticas a nivel nacional ${ }^{33}$.

\subsection{Función pública}

En cuanto a la función pública, mencionamos que está relacionada con la legitimidad y la efectividad de las decisiones, así como con la búsqueda del interés común.

Tal función se encuentra estrechamente relacionada con la posibilidad de acceder a

\footnotetext{
${ }^{31}$ Pilar Moraga, «La definición de nuevos estándares en materia de participación ciudadana en el sistema de evaluación de impacto ambiental chileno», Revista Derecho del Estado, Universidad del Estado de Colombia, 38, enero-junio (2017): 191.

${ }^{32}$ Alejandro Rojas, Francisco Sabatini y Claudia Sepúlveda, «Conflictos Ambientales en Chile: aprendizajes y desafíos», Revista Ambiente y Desarrollo de CIPMA XIX, $\mathrm{n}^{\circ} 2$ (2003): 24.

${ }^{33}$ Es claro que la normativa sobre participación ciudadana no tiene ninguna intención de propiciar ese fortalecimiento de la sociedad civil, pero sin perjuicio de ello, creemos que dicha articulación sucede. Esto último, sin embargo, no pasa de ser una observación de estos autores en su experiencia, carente de una metodología consistente para ser sustentada.
} 
la información pública, toda vez que transparenta el proceso de toma de decisiones reforzando la confianza de la ciudadanía. Pero además, se encuentra ligada a la posibilidad de incidencia de la comunidad en la decisión final, en lo que no es fácil separar las funciones de la participación ciudadana.

Sobre esta función pública, cabe hacer una pequeña mención, pues el hecho de que la participación propenda a aumentar la legitimidad produce una paradoja. Mientras una buena participación aumenta los niveles de legitimidad, una que sea dirigida exclusiva y específicamente a ello tendrá un efecto contrario, al denotar un espíritu de manipulación de una herramienta que debiera estar puesta al servicio de la satisfacción de un derecho.

Tanto la Comisión Asesora Presidencial de reformas al SEIA como la Comisión Sindical Cívico Parlamentaria ${ }^{34}$ en la misma materia, concuerdan en los problemas de legitimidad a los que se enfrenta el SEIA, los que, por lo demás, se enmarcan en una creciente desconfianza de la ciudadanía hacia las instituciones en general. En esa línea, el reciente informe del Consejo Nacional de Participación Ciudadana es claro en indicar que:

"[...] existe una pérdida de legitimidad y representatividad de las instituciones y los actores políticos lo suficientemente profunda en su nivel y extendida en el tiempo como para plantear que el sistema institucional y sus actores se van volviendo ilegítimos y no representativos. Se requieren respuestas rápidas y significativas" ${ }^{\prime 35}$.

Las causas son múltiples, pero es posible señalar como uno de los precursores la falta de un enfoque participativo en nuestro sistema que comprenda que la participación ciudadana contribuye efectivamente a mejorar las decisiones. Siguiendo a Delamaza, ello implica entender que el espacio público no se circunscribe a lo regulado por el Estado, existiendo un espacio público no estatal en dónde no solo conviven intereses particulares sino que también es un espacio en el que se puede velar por el interés común ${ }^{36}$.

El Consejo Nacional de Participación Ciudadana, en este sentido ha diagnosticado que en Chile el modelo imperante es de toma de decisiones verticales y jerárquicas, excluyendo de ese modo la posibilidad de una participación ciudadana sustantiva ${ }^{37}$.Y ello, creemos, se debe a la noción de que los intereses que se pueden hacer presentes a raíz de la participación ciudadana no velan por el interés general, a diferencia de la Administración en quien recae el deber general de velar por el bien común, creyéndose, en esa lógica, que una decisión jerárquica tendrá mayores probabilidades de velar por ello.

No obstante lo anterior, el Estado de Derecho actual demanda extender el espacio público más allá de lo estatal e incorporar el principio cooperativo. Schmidt Assman, al respecto, señala que: "La práctica, en efecto, pone de manifiesto que la cooperación es condición o presupuesto para el funcionamiento del Estado. Sólo [sic] a través de la cooperación con la sociedad puede el Estado obtener la información que precisa para llevar a cabo su función directora y de liderazgo. Para ello no bastan los contactos puntuales entre ambas esferas. Más bien resultan necesarias fórmulas de actuación integradas y duraderas que

\footnotetext{
${ }^{34}$ La Comisión Sindical Ciudadana Parlamentaria (CSCP) es una comisión creada de forma alternativa a la Comisión Asesora Presidencial (CAP) sobre la modificación al SEIA (impulsada por la Presidenta de la República, Michelle Bachelet en su periodo 2014-2018), que incluye a diferentes ONGs ambientales, parlamentarios y los respectivos sindicatos del SEA y Superintendencia del Medio Ambiente.

${ }^{35}$ Consejo Nacional de Participación Ciudadana, Cit. Ant. (26), 28.

${ }^{36}$ Gonzalo Delamaza, Cit. Ant. (5), 49.

${ }^{37}$ Consejo Nacional de Participación Ciudadana, Cit. Ant. (26), 30.
} 
Costa y Belemmi $\bullet$ ¿Susurros al viento? Desempeño de la participación ciudadana en el SEIA $・$ Sección doctrina

permitan identificar problemas e idear soluciones de forma conjunta" ${ }^{93}$.

Lo anterior es especialmente revelador frente a los conflictos ambientales, en los que no es posible para la Administración recopilar toda la información necesaria para, por ejemplo, dimensionar las externalidades negativas y positivas que la aprobación de un proyecto tendrá en una comunidad determinada, independiente de la capacidad técnica que tengan.

En ese sentido, es posible sostener que la práctica de la participación ciudadana al interior del SEIA, no ha incorporado completamente el principio de cooperación, toda vez que la configuración del sistema en lugar de ver a la ciudadanía como un aliado, suele posicionarla como una antagonista a los proyectos.

En este sentido nos parece ejemplificador mencionar dos situaciones: i) lo sucedido a raíz de la solicitud de apertura de participación ciudadana frente a proyectos presentados por DIA y ii) la interpretación que el SEA ha otorgado a la posibilidad de reclamar administrativa y judicialmente por no haberse considerado debidamente las observaciones ciudadanas en el procedimiento de evaluación.

Respecto a lo primero, como se adelantó, uno de los importantes avances que implicó la Ley $\mathrm{N}^{\circ} 20.417$ fue que incorporó la posibilidad de abrir un procedimiento de PAC frente a los proyectos ingresados por DIA, en su artículo 30 bis. Lo anterior con la limitación de que: i) el proyecto debe generar cargas ambientales; ii) debe ser solicitada por escrito dentro de los 10 días de publicado el ingreso del proyecto al SEIA en el Diario Oficial; y iii) para que el procedimiento se abra es necesario que lo soliciten al menos dos organizaciones ciudadanas con personalidad jurídica o como mínimo diez personas naturales directamente afectadas ${ }^{39}$.

Esta norma, que venía a solucionar el problema identificado respecto de la versión original de la Ley $\mathrm{N}^{\circ} 19.300$, en la práctica ha devenido en una barrera que dificulta que la comunidad se organice y pueda ejercer su derecho a participar. La interpretación del artículo 30 bis ha sido de las más conflictivas respecto a dos cuestiones centrales: i) la discrecionalidad que tiene el Servicio para decidir abrir o no el procedimiento y ii) cuándo se entiende configurado el requisito de generar cargas ambientales.

Respecto a la discrecionalidad del Servicio, la utilización del vocablo podrá, ha llevado a que el SEA interprete que frente a la solicitud posee una facultad discrecional para decidir si se abre o no un procedimiento PAC, independientemente de si se cumple con el requisito de ser solicitada por al menos 10 personas naturales directamente afectadas o dos organizaciones ciudadanas.

En ese sentido, el problema se ha dado porque el artículo 30 bis en su inciso penúltimo expresa que se debe entender que los proyectos producen cargas ambientales cuando "generan beneficios sociales y que ocasionan externalidades ambientales negativas en localidades próximas durante su construcción u operación" ${ }^{40}$. Al poco tiempo de publicadas las

\footnotetext{
${ }^{38}$ Eberhard Schmidt-Assmann, La teoría general del Derecho Administrativo como sistema. Objeto y fundamentos de la construcción sistemática (Madrid: Marcial Ponds, 2003): 39.

${ }^{39}$ Ley N ${ }^{\circ} 19.300$, sobre Bases Generales del Medio Ambiente, 9 de marzo de 1994, y sus posteriores modificaciones, artículo 30 bis.

${ }^{40}$ En específico el inciso penúltimo del artículo 94 del Reglamento del SEIA indica que "Se considera que generan cargas ambientales los proyectos o actividades cuyas tipologías correspondan a las letras a.1, b), c), d), e), f), j) y o) del artículo 3 de este Reglamento o que contengan partes, obras o acciones a las que apliquen
} 
reformas de la Ley $N^{\circ} 20.417$ Costa y Fuentes señalaban que "salvo una difícil interpretación restrictiva de los beneficios sociales, tenemos como consecuencia lógica que la inmensa mayoría de los proyectos que se someterán al SEIA tendrán esta característica"41.

Es del caso que esa "difícil interpretación restrictiva" fue la que tomó el SEA. Analizada así es que el Servicio ha rechazado la apertura de procedimientos de participación ciudadana en las DIAs por considerar que no se cumple con que los proyectos generen beneficios sociales, y existiendo solo externalidades negativas considera que la facultad que le entrega la Ley para abrir o no el procedimiento le permite rechazar las solicitudes ${ }^{42}$.

A modo de ejemplo, en el caso suscitado a raíz del proyecto "Incorporación de Tronadura como Método Complementario en la Extracción Mecánica de Material Estéril en Mina Invierno", el SEA de la Región de Magallanes rechazó las solicitudes de apertura de PAC, por considerar que no existían beneficios sociales asociados al proyecto, pero además sosteniendo que "si bien el proyecto genera externalidades negativas indicadas por los/as solicitantes, no corresponde a ninguna de las tipologías señaladas en el artículo 94 del RSEIA que generan cargas ambientales" ${ }^{\text {43 }}$. Es decir, se interpretó que solo los proyectos enumerados en el artículo 94 del Reglamento son los que generan cargas ambientales dejando fuera cualquier otro ${ }^{44}$.

La discusión sobre la interpretación del artículo 30 bis aún se encuentra abierta, sin embargo, respecto al mismo proyecto antes mencionado, la Excelentísima Corte Suprema dio luces respecto a su interpretación en la causa Rol 55.203-2016, en la que revirtió la decisión de no abrir el procedimiento PAC por considerar que el proyecto sí considera beneficios sociales y que el artículo 94 no establece un listado taxativo de proyectos que pueden generar cargas ambientales. En este sentido, el fallo, haciendo una revisión de la historia de la Ley $\mathrm{N}^{\circ} 20.417$, en que se señala expresamente que la idea es otorgar participación ciudadana a los proyectos que generan un impacto o carga negativa para la ciudadanía, reinterpretó las normas de modo que no se restringiera el principio participativo ambiental ${ }^{45}$.

Respecto a lo segundo, es decir, a la interpretación que el SEA ha otorgado a la posibilidad de reclamar administrativa y judicialmente por indebida consideración de las observaciones ciudadanas en el procedimiento de evaluación, el SEA ha sostenido reiteradamente, que no es posible reclamar ni administrativa ni judicialmente, sobre puntos que no se hayan tocado expresamente en la observación ciudadana realizada al interior del proce-

dichas tipologías, así como cualquier otro proyecto o actividad cuyo objetivo consista en satisfacer necesidades básicas de la comunidad, tales como proyectos de saneamiento, agua potable, energía, entre otros".

${ }^{41}$ Ezio Costa y Paula Fuente, «Participación ciudadana en las declaraciones de impacto ambiental», Revista de Derecho Ambiental III (2011): 99.

${ }^{42}$ Dicha interpretación ha sido sostenida, por ejemplo, en los procedimientos de evaluación de los proyectos Incorporación de Tronadura como Método Complementario en la Extracción Mecánica de Material Estéril en Mina Invierno, Potimización Planta Seleccionadora de Semillas y Centro de Cultivo Noroeste de Cobquecura.

${ }^{43}$ Servicio de Evaluación Ambiental, Resolución $\mathrm{N}^{\circ} 010 / 2016$ de fecha 12 de enero de 2016, considerando $\mathrm{N}^{\circ}$ 8: "Rechaza solicitud de participación ciudadana en Declaración de Impacto Ambiental: Incorporación de Tronadura como método complementario en la extracción mecánica de material estéril en Mina Invierno”.

${ }^{44}$ El artículo 94 contiene una lista de tipos de proyectos que en cualquier caso se comprenderá que generan cargas ambientales. En una versión previa del Reglamento del SEIA, se incluía en el artículo 94 el vocablo "únicamente", previo a la lista de los proyectos que producirían cargas ambientales. Sin embargo, dicha expresión se quitó en la posterior modificación del reglamento.

${ }^{45}$ Corte Suprema, Rol 55.203-2016, 16 de marzo de 2017, Considerando Noveno y Décimo tercero. 
Costa y Belemmi $\bullet$ ¿Susurros al viento? Desempeño de la participación ciudadana en el SEIA $・$ Sección doctrina

dimiento PAC, invocando el principio de congruencia. De acuerdo a esta interpretación, incluso la competencia del Tribunal Ambiental para conocer de las reclamaciones por observaciones ciudadanas quedaría circunscrita a lo observado en la primera etapa de evaluación del proyecto, incluso antes de dictada la $\mathrm{RCA}^{46}$.

Nuevamente, ha sido la jurisprudencia de los Tribunales Ambientales de Santiago y Valdivia, en fallos recientes del año $2017^{47}$, la que ha rechazado la interpretación del SEA, ampliando el abanico de posibilidades para los observantes PAC que desean reclamar, señalando que es posible hacerlo sobre todas aquellas materias que tengan relación con lo observado, sin circunscribir la reclamación a lo específicamente señalado en la observación realizada en la instancia de participación ciudadana del SEIA ${ }^{48}$. Muestra de ello es el fallo del Tercer Tribunal Ambiental, en la causa Rol R-47-2017, en la que indicó que la inalterabilidad de la pretensión no puede ser estricta, y que se debe considerar que las observaciones, la mayoría de las veces, se realizan sin el patrocinio de un abogado ${ }^{49}$. Además señaló que la observación al interior del SEIA no tiene una pretensión anulatoria sino que "su objeto es advertir a la Administración para que tenga en cuenta aspectos que los participantes estiman relevantes desde e1 punto de vista ambiental ${ }^{, 50}$. Y que solo una vez notificada la RCA se puede definir cuál es la pretensión. Finalmente, agrega en su Considerando Décimo que: "el Comité de Ministros no puede extender su decisión a aspectos de la evaluación ambiental que no guarden relación alguna con lo comentado durante la PAC por ese participante específico, no obstante no exigirá que exista una coherencia estricta entre lo solicitado en el recurso administrativo de reclamación y la PAC, ni tampoco entre el recurso judicial de reclamación y el recurso administrativo de reclamación. Siendo así, el Comité de Ministros deberá resolver todos los aspectos de forma y fondo que reclamen los participantes en la PAC, siempre que exista una conexión significativa entre los hechos alegados en la PAC y en el recurso administrativo. Este Tribunal a su vez, conocerá de la reclamación judicial siempre que guarde una conexión significativa entre los hechos alegados en el recurso administrativo y en el judicial, sin perjuicio que las alegaciones jurídicas puedan variar siempre que se mantenga intacta la pretensión anulatoria".

Como se puede apreciar, el SEA, organismo en quien recae el deber de promover la participación ciudadana, ha optado por interpretar las normas que permiten incorporar la participación ciudadana a los procesos comenzados por DIA y las que permiten realizar reclamaciones en razón de observaciones ciudadanas, de una forma que la restringe, sin constatar que la interpretación contraria ayudaría a mejorar sus decisiones y a alcanzar mayor legitimidad de las mismas. Por otro lado, ha sido la jurisprudencia, la que ha otorgado luces de una interpretación que responde a las necesidades de la comunidad de participar en la decisión administrativa, para cumplir con el objetivo de mejorar las decisiones ambienta-

\footnotetext{
${ }^{46}$ En este sentido es posible citar los casos referidos a los proyectos: Candelaria 2030-Continuidad operacional (RCA del año 2015), Parque Eólico Chiloé (RCA del año 2015) y Hotel Punta Piqueros (RCA del año 2014).

${ }^{47}$ Ver: Tercer Tribunal Ambiental, R-47-2017, de 21 de septiembre de 2017 y Segundo Tribunal Ambiental, R-131-2016, de 28 de abril de 2017.

${ }^{48}$ Se debe precisar de todos modos que ninguna de las reclamaciones fue acogida.

${ }^{49}$ Tercer Tribunal Ambiental, Rol R-47-2017, de 21 de septiembre de 2017, Considerando Séptimo.

${ }^{50}$ Tercer Tribunal Ambiental, Cit. Ant., (49), Considerando Octavo.
} 
les y de ese modo otorgar mayor legitimidad a la decisión pública.

\subsection{Función privada}

Por función privada de la participación entendemos su capacidad de dar cuenta de los intereses particulares que existen en juego y lograr acuerdos.

Por una parte, el sistema de reclamaciones consagrado en los artículos 20 y 29 de la Ley $\mathrm{N}^{\circ} 19.300$, en relación con la Ley $\mathrm{N}^{\circ} 20.600$, puede ser visto como una manifestación de esta función privada, en el sentido de que se permite la reclamación administrativa y judicial en contra de las RCA, a aquellas personas cuyas observaciones no hayan sido adecuadamente consideradas. No existe, a priori, una causal de reclamación que tenga relación con intereses públicos o comunitarios, más allá del contenido que tenga la propia observación y sin perjuicio de la potestad del Comité de Ministros, el Director Ejecutivo del SEA y los Tribunales Ambientales, de revisar ampliamente la legalidad de las resoluciones.

Vista así, la participación ciudadana pareciera un requisito meramente formal de legitimación activa para reclamar respecto de las RCA, dando cuenta de que son, principalmente, los intereses privados los que se tuvieron en cuenta al momento de regular sobre el sistema recursivo en base las observaciones ciudadanas.

Por otra parte, la manifestación más clara de esta función en la Ley $\mathrm{N}^{\circ} 19.300$ es el reconocimiento de la existencia de negociaciones entre el titular del proyecto y la comunidad afectada en su artículo 13 bis, el que establece la obligación de informar al SEA tales negociaciones. Desde esta perspectiva existe la posibilidad de llegar a acuerdos y de que estos sean reconocidos, relevando los intereses particulares existentes al interior del SEIA.

No obstante lo anterior, el reconocimiento de negociaciones previas no se hace a instancias del SEIA, por lo que no se trata de una circunstancia regulada. Ello ha generado críticas, precisamente por la situación desigual en la que se encuentra la comunidad que no conoce el proyecto (independientemente del resguardo tomado por el mismo artículo 13 bis en cuanto a que tales acuerdos deben ser informados y no serán vinculantes para la calificación ambiental del proyecto o actividad). Al respecto, el Informe Evaluaciones de Desempeño Ambiental de Chile del año 2016, recomienda: "Perfeccionar el proceso de realización de evaluaciones de impacto ambiental, con el fin de asegurar que se tomen seriamente en consideración proyectos alternativos, se garantice la participación ciudadana en las etapas preliminares y se otorgue más atención a los efectos ambientales potenciales, especialmente en los ecosistemas", 51 .

Ello porque diagnóstica que la participación ciudadana al interior del SEIA se da en etapas muy avanzadas del proyecto, relegando los posibles aportes a la mejora de las medidas de mitigación, sin tener incidencia en el diseño del proyecto ${ }^{52}$. Lo anterior resulta especialmente atendible si se considera que la incorporación de la posibilidad de abrir una etapa de participación ciudadana por modificaciones sustanciales, se basaba en el mismo diagnóstico y que ya en el año 2003, Rojas, Sabatini y Sepúlveda nos indicaban que "[...] el SEIA presenta serias limitaciones a la participación. No contempla la evaluación de alternativas, y solo incluye la participación ciudadana cuando el estudio de impacto ambiental del res-

\footnotetext{
${ }^{51}$ OCDE-CEPAL, Cit. Ant. (12), 31.

${ }^{52}$ OCDE-CEPAL, Cit. Ant. (12), 119.
} 
Costa y Belemmi $\bullet$ ¿Susurros al viento? Desempeño de la participación ciudadana en el SEIA $・$ Sección doctrina

pectivo proyecto ha sido concluido y está listo para ser evaluado. A pesar de ello para muchas empresas la evaluación ambiental de los proyectos y, sobre todo, la participación ciudadana son verdaderas trabas a la inversión. Responder a estas exigencias legales representó para ellas un desafío mayor" ${ }^{25}$.

En esta línea, actualmente se pronunció la Comisión Asesora Presidencial, proponiendo una etapa de relacionamiento temprano entre las comunidades y el titular del proyecto, mediado por el SEA, que permita que la comunidad tome conocimiento del proyecto y pueda tener incidencia en la etapa de diseño del mismo.

Ahora bien, vemos con preocupación que tal iniciativa, siguen estando enfocada desde los intereses privados de los actores, suponiendo una posibilidad de negociación que no pareciera tener la potencialidad de mejorar las relaciones y disminuir los conflictos, en cuanto ellos no son solo relacionados con intereses particulares, sino generalmente también con intereses comunitarios o públicos.

Toda aproximación que se plantee, solo será un avance en el cumplimiento de los objetivos de la participación ciudadana, siempre y cuando esté mediada por asesoría técnica adecuada para la comunidad. Solo así podría generar instancias más propicias para lograr acuerdos con ella (que no obsten a la participación ciudadana) ${ }^{54}$ y que avancen, no solo en el reforzamiento de la función privada, sino también de la función pública y comunitaria, incluyendo de manera efectiva las inquietudes de la comunidad en la decisión ambiental.

\section{Conclusiones}

Hemos revisado la participación ciudadana en el SEIA, con el objeto de evaluar si la manera en que ella se ha construido y opera, está en línea con las funciones que la literatura y las propias normas de participación le han atribuido.

Para realizar lo anterior nos basamos en las funciones que la Carta Iberoamericana de Participación en la Gestión Pública expresa, agrupándolas en tres categorías: i) Función Comunitaria; ii) Función Pública; y iii) Función Privada y nos hemos valido, principalmente, de las normas relativas a la participación ciudadana en el SEIA, los instructivos del SEA y los fallos judiciales que se han referido al tema.

De lo revisado concluimos que, en general la participación ciudadana en el SEIA no se ha desarrollado con miras a servir a las funciones que teóricamente se le han entregado, existiendo en diversas instancias barreras artificiales que complejizan la satisfacción de un derecho que de por sí es difícil de ejercer y garantizar. En este sentido, respecto de la fun-

\footnotetext{
${ }^{53}$ Alejandro Rojas et al., Cit. Ant. (32), 23.

${ }^{54}$ Cabe consignar que desde el año 2013 el SEA tiene una "Guía para la participación anticipada de la comunidad en proyectos que se presentan al SEIA” en la que se expresan las virtudes de generar un acercamiento entre la comunidad y el titular de forma previa a la presentación del proyecto, pues de esa forma "será más fácil reconocer las inquietudes y deseos de cada parte, de modo de poder alcanzar acuerdos y consensos de mutuo beneficio". El problema de ella es que es un instrumento indicativo, toda vez que el procedimiento que reconoce no se encuentra al interior de la institucionalidad, siendo discrecional cumplir o no con un procedimiento adecuado de acercamiento a la comunidad. Ver: http://www.sea.gob.cl/sites/default/files/migration_files/guias/GuiaPAC_anticipada_FINAL_20130711.pdf
} 
ción comunitaria la Ley $\mathrm{N}^{\circ} 20.417$ significó importantes avances, sin embargo, aún quedan materias pendientes, siendo ejemplo de ello i) la indefinición de la Ley respecto a la "debida consideración de las observaciones ciudadanas" y ii) las asimetrías de información existentes entre la comunidad, Administración y titular.

Por su parte, sobre la función pública se constató una crisis de legitimidad en las decisiones tomadas al interior del SEIA, debido a la desconfianza generalizada en las instituciones, y en particular, por no considerar la importancia de la colaboración de la ciudadanía en la toma de decisiones ambientales, identificándose como ejemplo de ello, la interpretación restrictiva que el SEA ha realizado respecto de la posibilidad de abrir un procedimiento de PAC en los proyectos presentados mediante DIA y respecto a la relación que debe existir entre lo observado y la posible reclamación PAC que se presenta ante el Comité de Ministros y los Tribunales Ambientales.

Por último, la única de las funciones que está recogida de alguna manera por la normativa, es la función privada. Sin embargo, incluso esta última termina siendo consagrada al interior del SEIA solo en una etapa posterior a la propia participación, de suerte de entregar una herramienta recursiva a los observantes, que no necesariamente se condice con los objetivos del SEIA ni de la regulación ambiental.

El hecho de que la participación sea construida principalmente desde esta función privada y contradictoria, claramente aumenta la posibilidad de que se produzcan conflictos socio-ambientales, pues obvia cualquier potencialidad deliberativa de la herramienta, además de vulnerar abiertamente los estándares sobre los que se construye el derecho a la participación.

No obstante lo anterior, de lo revisado se evidencia que la reciente jurisprudencia ha contribuido a morigerar la interpretación restrictiva del SEA, inclinándose por otorgar importancia central al principio de participación ciudadana y por realizar una interpretación favorable al desarrollo de la misma. Sin embargo, aún queda camino para poder sostener que se cumple correctamente con los estándares de participación ciudadana como derecho.

En este camino, en primer lugar, al menos debiera reconocerse la obligación del SEA de interpretar las normas en la manera en que ellas permitan mayor y mejor participación, en razón del deber general del Estado establecido en el artículo 4 de la Ley $\mathrm{N}^{\circ} 19.300$ y del deber particular establecido en el reglamento del SEIA. En seguida, la comprensión e interpretación de los mecanismos de participación ciudadana desde las funciones que ellos debieran cumplir, debería implicar una apertura hacia espacios de mayor deliberación en que todos los actores involucrados puedan contribuir de manera conjunta y con ciertos niveles de igualdad mínimas que garanticen esa deliberación.

\section{Bibliografía}

Libros y artículos

Aguilar, Gonzalo. Gobierno Ambiental por consentimiento, participación y evaluación ambiental. En: Evaluación medioambiental, participación y protección del medio ambiente. Santiago: Librotecnia, 2013.

Astorga, Eduardo. Derecho Ambiental Chileno. Santiago: Lexis Nexis, 2006.

Biblioteca del Congreso Nacional. Historia de la Ley 19.300. Valparaíso, 1993. 
Costa y Belemmi • ¿Susurros al viento? Desempeño de la participación ciudadana en el SEIA • Sección doctrina

Castellá, Josep. Los derechos constitucionales de participación política en la Administración Pública. Barcelona: Cedecs, 2011.

CEPAL. Acceso a la información, participación y justicia en temas ambientales en América Latina y el Caribe. Situación actual, perspectivas y ejemplos de buenas prácticas. Serie Medio Ambiente y Desarrollo $\quad N^{\circ} \quad 151 \quad$ (Santiago, 2013), http://www.cepal.org/publicaciones/xml/9/51389/Accesoalainformacion.pdf , acceso el 31 de agosto de 2017.

CLAD. Carta Iberoamericana de participación ciudadana en la gestión Pública (Lisboa: 2009).

Consejo Nacional de Participación Ciudadana. Estado de la participación ciudadana en Chile y propuestas de reforma a la Ley 20.500 sobre Asociaciones y Participación Ciudadana en la Gestión Pública (Santiago: Enero 2017), http://consejoparticipacion.cl/content/uploads/2017/01/informe-final.pdf , acceso el 30 agosto 2017. Costa, Ezio y Fuente, Paula. «Participación ciudadana en las declaraciones de impacto ambiental», Revista de Derecho Ambiental III (2011): 83-106.

Delamaza, Gonzalo. «Espacio público y participación ciudadana en la gestión pública en Chile: Límites y Posibilidades», Polis. Universidad Bolivariana, vol. $10 \mathrm{~N}^{\circ} 30$ (2011).

Hervé, Dominique. Justicia Ambiental y Recursos Naturales. Valparaíso: Ediciones Pontificia Universidad Católica de Valparaíso, 2015.

Larios, María Jesús. La participación ciudadana en la elaboración de la ley. Madrid: Congreso de los diputados, 2003.

Ministerio del Medio Ambiente. Comisión Asesora Presidencial para la Evaluación del SEIA. Informe Final (Santiago: Junio 2016), http://portal.mma.gob.cl/wp-content/doc/35877 InformeMMAF_FINAL.pdf, acceso el 31 agosto 2017.

Mirosevic, Camilo. «La participación ciudadana en el procedimiento de evaluación de impacto ambiental y las reformas introducidas por la Ley No 20.417», Revista de Derecho de la Pontificia Universidad Católica de Valparaíso 36 (2011): 281-323.

Moraga, Pilar. «La definición de nuevos estándares en materia de participación ciudadana en el sistema de evaluación de impacto ambiental chileno», Revista Derecho del Estado, Universidad del Estado de Colombia, 38, enero-junio (2017): 177-198.

OECD, Focus on Citizens: Public Engagement for Better Policy and Services (París, 2009), http://www20.iadb.org/intal/catalogo/pe/2009/03785.pdf , acceso el 31 agosto 2017, 17.

OCDE-CEPAL, Evaluación de Desempeño Ambiental (Santiago, 2005), http://repositorio.cepal.org/bitstream/handle/11362/1288/S0500003 es.pdf;jsessionid=20A3F661E B0CE290E3F449ECDC1BC7CB? sequence=1, acceso el 30 de agosto de 2017.

OCDE-CEPAL. Evaluación del Desempeño Ambiental de Chile. Santiago: 2016, http://repositorio.cepal.org/bitstream/handle/11362/40308/S1600413_es.pdf , acceso el 31 de agosto de 2017.

Rojas, Alejandro et. al. «Conflictos Ambientales en Chile: aprendizajes y desafíos», Revista Ambiente y Desarrollo de CIPMA XIX, $\mathrm{n}^{\circ} 2$ (2003): 22-30.

Eberhard Schmidt-Assmann. La teoría general del Derecho Administrativo como sistema. Objeto y fundamentos de la construcción sistemática. Madrid: Marcial Ponds, 2003.

Normas e instructivos 
Ley $N^{\circ}$ 19.300, sobre Bases Generales del Medio Ambiente, 9 de marzo de 1993.

Ley $\mathrm{N}^{\circ}$ 20.417, Crea el Ministerio, el Servicio de Evaluación Ambiental y la Superintendencia del Medio Ambiente, de 26 de enero de 2010.

Decreto $N^{\circ} 40$, Ministerio del Medio Ambiente, Aprueba Reglamento del Sistema de Evaluación de Impacto Ambiental, de 12 de agosto de 2013.

Servicio de Evaluación Ambiental. Oficio Ordinario $N^{\circ}$ 100.142/2010. Instruye sobre la admisibilidad de observaciones ciudadanas en los procesos de participación ciudadana en el SEIA.

Servicio de Evaluación Ambiental. Oficio Ordinario $N^{\circ} 130.528 / 2013$. Imparte instrucciones sobre la consideración de las observaciones ciudadanas en el marco del procedimiento de evaluación de impacto ambiental.

Jurisprudencia judicial

Corte Suprema, Rol 55.203-2016, 16 de marzo de 2017.

Segundo Tribunal Ambiental, Rol R-131-2016, de 28 de abril del año 2017.

Segundo Tribunal Ambiental, Rol R-101-2016, de 31 de mayo de 2017.

Segundo Tribunal Ambiental, Rol R-35-2014, 18 de febrero de 2016.

Tercer Tribunal Ambiental, R-47-2017, de 21 de septiembre de 2017.

Recibido: 31-08-2017

Aceptado: 9-11-2017

Filiación institucional de los autores:

Ezio Costa, Investigador del Centro de Regulación y Competencia (Regcom), Facultad de Derecho, Universidad de Chile.

Victoria Belemmi, Investigadora ONG Fima. 